\title{
Expression of ribophorine II is a promising prognostic factor in human gastric adenocarcinoma
}

\author{
DAISUKE FUJIMOTO, TAKANORI GOI and YASUO HIRONO \\ Department of Surgery 1, Faculty of Medicine, University of Fukui, Fukui 910-1193, Japan
}

Received October 17, 2016; Accepted December 19, 2016

DOI: $10.3892 /$ ijo.2016.3822

\begin{abstract}
The increased invasiveness of gastric adenocarcinoma is important for progression and metastasis. In recent molecular biological studies, ribophorine II (RPN2) induced epithelial-mesenchymal transition and metastatic activity. However, no studies have evaluated the relationship between RPN2 expression, ability of cancer to invade/metastasis, and patient prognosis in gastric adenocarcinoma. Therefore, we have examined these factors. Immunohistochemical staining was performed to detect RPN2 and p53 in the primary lesion and adjacent normal gastric mucosa of 242 gastric adenocarcinoma patients who underwent resection surgery. We conducted clinicopathologic examinations and analyzed patient prognoses with the Kaplan-Meier method. Further, multivariate analysis was conducted using a Cox hazard model. Also, we analyzed the ability of invasion under inhibited RPN2 expression in vitro. RPN2 expression was observed in 119 of 242 cases of gastric adenocarcinoma patients. RPN2 expression was associated with a higher incidence of depth of wall invasion, lymph node metastasis, lymphatic invasion, venous invasion, peritoneal dissemination, histopathological stage, and p53 expression. In stage II and III curative resection cases, where recurrence is the most serious problem, cases that expressed RPN2 had a significantly lower 5-year survival rate and higher recurrence rate compared to the cases with no RPN2 expression. In the multivariate analysis for prognosis, RPN2 expression was found to be an independent factor. Also, gastric adenocarcinoma cell, had mutant-type p53, reduced the ability of invasion by knockout of RPN2 expression in vitro. RPN2 expression correlates with gastric adenocarcinoma cell invasion and shows promise as a new prognostic factor in human gastric adenocarcinoma.
\end{abstract}

Correspondence to: Dr Daisuke Fujimoto, Department of Surgery 1, Faculty of Medicine, University of Fukui, 23-3 Matsuoka Shimoaiduki, Eiheiji-cho, Yoshida-gun, Fukui 910-1193, Japan

E-mail: fujimoto@u-fukui.ac.jp

Key words: ribophorine II, gastric adenocarcinoma, prognostic factor, CRISPR/Cas9, immunohistochemistry

\section{Introduction}

Gastric adenocarcinoma has one of the highest prevalence and mortality rates among malignant tumors (1). Global incidence of primary tumor locations and the histological types are constantly changing: in United States and in Western Europe the incidence of Barrett's type carcinoma and gastric cardia adenocarcinoma is increasing (2), while there has been a reduction of incidence of distal gastric adenocarcinoma since the 1970s (3). Although gastric adenocarcinoma mortality has been reduced, it remains a disease with poor prognosis and high mortality, second only to lung tumor in the world. The prognosis of gastric adenocarcinoma depends on stage and signature metastasis type of peritoneal dissemination have poor prognosis and when the disease is confined to the stomach mucosa, 5-year survival is $~ 95 \%$, while the reported 5-year survival rate for advanced gastric adenocarcinoma with peritoneal dissemination varies from 10 to $20 \%$ (3).

Peritoneal dissemination of gastric adenocarcinoma is particularly frequent, driving the need for therapies that minimize the cancer spread. One model for the mechanism of peritoneal metastasis of gastric adenocarcinoma involves growth of tumor at the primary lesion, invasion into serous surface, expose abdominal cavity, dissociation of cancer cells, and implantation and growth on the peritoneum. Various genetic alterations have been studied, and involvement of a number of genes has been reported.

MicroRNA (miRNA) is a small non-coding RNA that functions in RNAs silencing and post-transcriptional regulation of gene expression (4-6). These are the fundamental gene regulators that control proliferation, differentiation, and apoptosis during development. They target a large number of mRNAs and induce mRNA degradation or inhibition of translation by targeting the 30-untranslated regions (5). Some miRNAs had controlled the peritoneal dissemination of gastric adenocarcinoma (7-9). miR-27 promoted epithelial-mesenchymal transition (EMT) and metastasis of human gastric cancer cells (10). EMT is a portmanteau concept that can be applied to the metastatic behavior of carcinoma cells at a number of junctures, and has been associated with peritoneal dissemination of gastric adenocarcinoma (11). Various genetic alterations associated with EMT have been studied, and involvement of a number of genes has been reported. Ribopholin II (RPN2) is one of the 
genes that promote EMT through the stabilization of mutant p53 (12), and there is a possibility that miRNA is controlling RPN2. However, in gastric adenocarcinoma, no report exists on the relation between RPN2 and metastasis and prognosis of gastric adenocarcinoma.

This study was conducted to compare the expression of RPN2 with the invasiveness of the primary lesion in human gastric adenocarcinoma, and the clinicopathological features and patient prognosis, as reported below.

\section{Materials and methods}

Patients and samples. Surgical specimens and adjacent normal gastric tissues were obtained from 242 patients with sporadic primary gastric adenocarcinoma, and surgically resected in the Department of First Surgery, University of Fukui, Japan, between 2002 and 2010. As histopathological findings varied within the same tumors, the diagnosis was based upon the dominant pattern evaluated by two pathologists. All samples were fixed in $10 \%$ paraformaldehyde $(\mathrm{pH} 6.8)$ for $24 \mathrm{~h}$, and embedded in paraffin.

The eligibility criteria were as follows: i) histopathologically confirmed primary gastric adenocarcinoma, ii) resection of gastric adenocarcinoma with extended (D1+ or D2) lymph node dissection (13), iii) pathological diagnosis for the classification used the 7th edition UICC TNM classification, iv) histological curative resection (stage I-III), v) an Eastern Cooperative Oncology Group performance status of 0 or 1 , vi) no chemotherapy or radiotherapy before surgical resection, vii) patients with stage II/III received S-1 after surgical resection, viii) patients with stage IV received $\mathrm{S}-1$ + cisplatin after surgical resection, ix) patients with stage I received no chemotherapy after surgical resection, and x) all patients were followed up for recurrence at regular intervals for five years, underwent chest X-ray, computed tomography, and testing of gastrointestinal fiber. All the patients provided written informed consent before the samples were collected.

Ethical approval. We attest that the research was performed in accordance with the humane and ethical rules for human experimentation that are stated in the Helsinki Declaration of 1964 and latest version. The procedures of our study received ethical approval with Institutional Committee Responsible for Human Experimentation at University of Fukui and all those who participated in our study did so voluntary, having given their informed consent.

Immunohistochemical study. Paraffin sections, 4- $\mu$ m thick, were de-paraffinized with xylene and dehydrate through a grade ethanol series. Endogenous peroxidase activity was blocked by incubation for $30 \mathrm{~min}$ with $1 \%$ hydrogen peroxidase methanol. These hydrate sections were incubated in a dilution of skim milk powder for $30 \mathrm{~min}$ to reduce nonspecific staining, and incubated overnight with anti-RPN2 Ab (Aviva Systems Biology, CA, USA) or anti-p53 Ab (Dako, Denmark) at $4^{\circ} \mathrm{C}$ in a humidified chamber. After washing with Tris-buffered saline (TBS) buffer, and analyzed for the expression of RPN2 protein or $\mathrm{p} 53$ protein by the ChemMate method (Dako). The sections were developed with activated 3'-diami- nobenzidinetetrahydrochloride for $5 \mathrm{~min}$ and the reaction was stopped in TBS. Finally, the slides were lightly counterstained with hematoxylin. The expression was interpreted as positive when the protein was expressed in $>20 \%$ of the cancer cells using ImageJ software (http://rsb.info.nih.gov/ij/).

Statistical analysis. Statistically significant differences in clinicopathological findings were assessed by cross-tabulation, and statistical evaluations were determined by the $\chi^{2}$ test using SPSS software (IBM SPSS Statistics, IBM Corp., USA).

Patient survival was calculated using the Kaplan-Meier technique. The outcomes from different groups of patients were compared by log-rank test using SPSS software. The Cox proportional hazards model was used in multivariate regression analysis of survival data using SPSS software. P-values $<0.05$ were considered statistically significant.

Cell culture and RT-PCR analysis. Human gastric cancer cell lines MKN45, MKN74, NUGC3, NUGC4, SNU5, KATO III and TMK1 (which were obtained from JCRB Cell Bank) were maintained in RPMI-1640 supplemented with 10\% FBS (Gibco), $100 \mathrm{U} / \mathrm{ml}$ penicillin, and $100 \mu \mathrm{g} / \mathrm{ml}$ streptomycin at $37^{\circ} \mathrm{C}$ with $5 \% \mathrm{CO}_{2}$ incubation. RNA was isolated from the above cells using Isogen (Wako, Japan), according to the manufacturer's instructions. cDNA was synthesized from $2 \mu \mathrm{g}$ of total RNA by reverse transcription, using High-Capacity cDNA Reverse Transcription kits (Applied Biosystems). The resulting cDNA was used for the subsequent PCR assays. RPN2 was amplified by using the primers with following sequences: forward, 5'-GCCAGGAAGTGGTGTTTGTT-3'; and reverse, 5'-ACAGAGCGAAGAGCAGAAGC-3'.

Cell transfection. MKN74 and KATO III cells were seeded onto 6-well plates (Corning) $24 \mathrm{~h}$ before transfection. Cells were transfected using Lipofectamine 3000 (Life Technologies) at $80-90 \%$ confluency according to the manufacturer's instructions. For each well of a 6 -well plate, a total of $1 \mu \mathrm{g}$ of sgRPN2 (single-guided RNA to RPN2) + pGuide-it-ZsGreen1 plasmid or empty plasmid was used. The CRISPR/Cas9 system used in this study was Guide-it CRISPR/Cas9 systems (Clonetch). We designed sequences targeting the RPN2 locus (Fig. 4A). The plasmid enhanced green fluorescent protein (eGFP) and was used as a fluorescent marker to sort transfected cells. Forty-eight hours posttransfection, cells were pelleted in PBS $+2 \%$ FBS and sorted in 96-well plates using fluorescenceactivated cell sorting (FACS) with a FACSAria II cell sorter (BD BioSciences). Single cells from two populations of GFP-expressing cells (high expression and medium expression) were expanded to obtain individual clones.

Detection of nuclease-induced mutations. Genomic DNA was extracted from cells with the Multisource Genomic DNA Miniprep kit (Axygen), and then amplified by PCR with forward primer 5'-GCCAGGAAGTGGTGTTTGTT-3' and reverse primer 5'-ACAGAGCGAAGAGCAGAAGC-3'. The PCR product was resolved in $1.5 \%$ agarose gel. Target bands were cut and purified with QIAEX II Gel Extraction kit (Qiagen). The purified PCR products were mixed with $2 \mu \mathrm{l}$ 10X T7E1 nuclease buffer and nuclease-free water to a volume of $19 \mu 1$. These products were denatured for $10 \mathrm{~min}$ at $95^{\circ} \mathrm{C}$, 
A

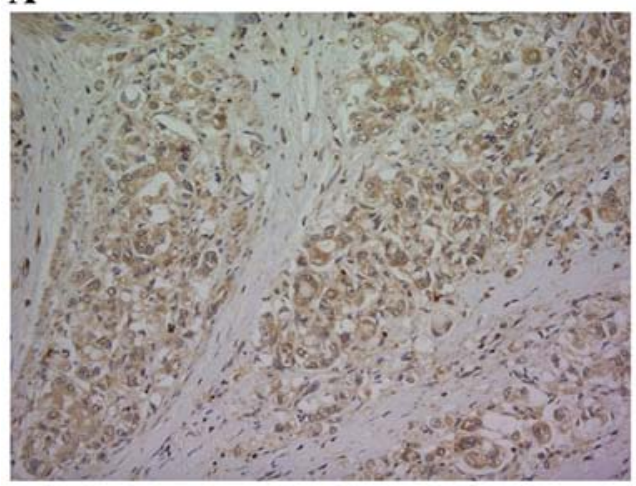

B

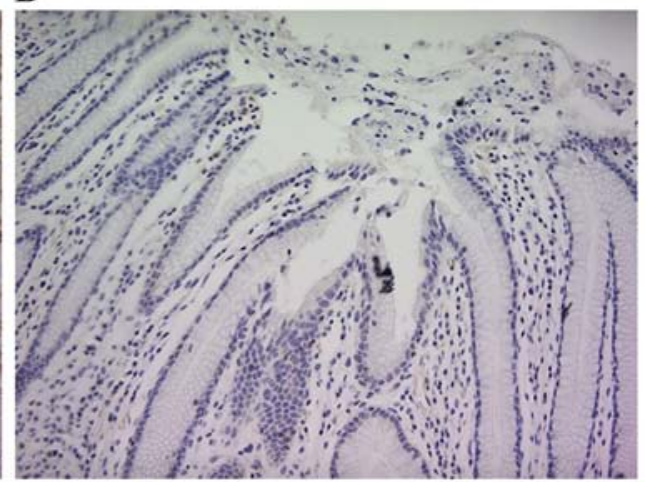

Figure 1. RPN2 protein expression in normal human gastric mucosa and primary gastric adenocarcinoma by immunohistochemical staining with antiRPN2 Ab. (A) RPN2 protein stained in brown was detected in the cytoplasm of primary gastric adenocarcinoma lesions. (B) RPN2 expression was not detected in normal human gastric mucosa.

annealed by gradual cooling in a thermocycler and digested by $1 \mu 1$ T7E1 nuclease (GeneCopoeia). The digestion was performed at $37^{\circ} \mathrm{C}$, in a water bath for $40 \mathrm{~min}$, and followed by analyzing in $1.5 \%$ agarose gel. Briefly, the gel was imaged and the intensity of the bands in each lane was measured by using ImageJ Software. For each lane, we calculated the fraction of the PCR product cleaved by using the following formula: fcut $=(B+C) /(A+B+C)$, where $A$ is the intensity of the undigested PCR product, while $B$ and $C$ are the intensities of each cleaved band. The Indel percentage was estimated by applying the following formula: Indel $(\%)=100 \times[1-\sqrt{ }(1-f c u t)]$.

Transfection of small interfering RNA. Small interfering RNA (siRNA) against RPN2 and control non-targeting siRNA were obtained from Invitrogen, Inc. (CA, USA). The non-silencing control siRNA, which has no sequence homology to any known human gene sequence, was used as a control for nonspecific effects in all experiments. Subconfluent MKN45 cells were transfected with siRNA using Lipofectamine 3000 transfection regent (Life Technologies) according to the manufacturer's instructions. Two days after transfection, the efficacy of siRNA knockdown was assessed using western blot analysis.

Western blot analysis. Total cell protein was extracted using RIPA buffer. Proteins in the lysate were resolved by SDS-PAGE using a 5-20\% SuperSep gel (Wako). The resolved proteins were transferred to nitrocellulose membrane. Protein bands were incubated with primary antibody overnight at $4^{\circ} \mathrm{C}$. Signals were visualized by enhanced chemiluminescence according to the manufacturer's instructions (GE Healthcare). Anti-RPN2 Ab was from Aviva Systems and anti-GAPDH Ab was from IMGENEX.

Cell invasion assay. This assay was performed using a cell invasion kit from Cell Biolabs, Inc. (CA, USA). Briefly, the invasion chambers were warmed up at room temperature for $10 \mathrm{~min}$, and the basement membrane layer was rehydrated with $300 \mu \mathrm{l}$ of warm serum-free media for $1 \mathrm{~h}$ at room temperature. After removing the rehydration medium from the inserts, $300 \mu 1$ of $1.0 \times 10^{6}$ cells $/ \mathrm{ml}$ in serum-free media was added to the inside of each insert, and $500 \mu \mathrm{l}$ of media containing

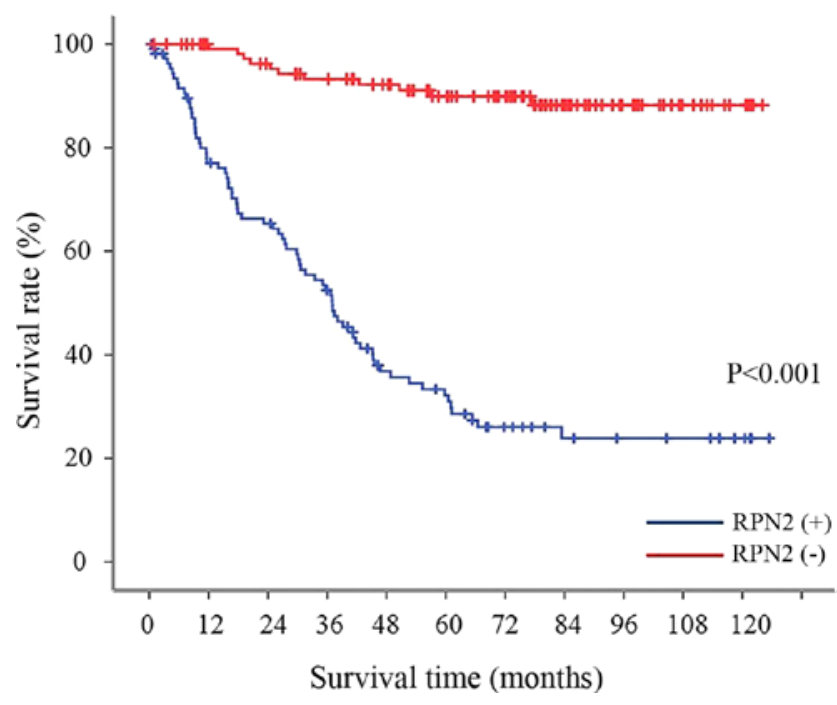

Figure 2. Relationship between RPN2 expression and survival rates in all human gastric adenocarcinoma patients. Patients with RPN2-positive tumors had significantly poorer prognosis than the patients with RPN2-negative tumors $(\mathrm{P}<0.001)$.

$10 \%$ FBS was added to the lower well of the invasion plate. The plate was then incubated at $5 \% \mathrm{CO}_{2}$ for $24 \mathrm{~h}$. The media was aspirated from the inside of the insert and the non-invasive cells were removed. The inserts were then transferred to clean wells containing $400 \mu \mathrm{l}$ of cell staining solution from the kit and were incubated for $10 \mathrm{~min}$ at room temperature. The stained inserts were washed several times with water and were air-dried. Inserts were transferred to new wells with $200 \mu \mathrm{l}$ of extraction solution from the kit and were incubated for $10 \mathrm{~min}$ with rotation. A volume of $100 \mu 1$ solution was used to measure the OD $560 \mathrm{~nm}$ in a spectrometer.

\section{Results}

RPN2 expression in human gastric adenocarcinoma. Although RPN2 expression was not observed in the normal gastric mucosal membrane adjacent to human gastric adenocarcinoma, its expression was intense in the primary lesion of gastric adenocarcinoma. Fig. 1 shows a respective representa- 
Table I. Correlation of RPN2 expression and clinicopathological findings.

\begin{tabular}{|c|c|c|c|}
\hline & & RPN2-positive & \\
\hline & No. of cases & No. of cases $(\%)$ & P-value \\
\hline All cases & 242 & $119(49.2)$ & \\
\hline Gender & & & 0.236 \\
\hline Male & 162 & $84(51.9)$ & \\
\hline Female & 80 & $35(43.8)$ & \\
\hline $\begin{array}{l}\text { Histological } \\
\text { type }\end{array}$ & & & 0.059 \\
\hline Pap, tub & 128 & $56(43.8)$ & \\
\hline Por, sig & 111 & $60(54.1)$ & \\
\hline Muc & 3 & $3(100)$ & \\
\hline $\begin{array}{l}\text { Depth of wall } \\
\text { invasion }\end{array}$ & & & $<0.001$ \\
\hline $\mathrm{T} 1$ & 79 & $13(16.5)$ & \\
\hline $\mathrm{T} 2$ & 30 & $17(56.7)$ & \\
\hline $\mathrm{T} 3$ & 26 & $15(57.7)$ & \\
\hline $\mathrm{T} 4$ & 107 & $74(69.2)$ & \\
\hline $\begin{array}{l}\text { Lymph node } \\
\text { metastasis }\end{array}$ & & & $<0.001$ \\
\hline No & 95 & $24(25.3)$ & \\
\hline N1-3 & 147 & $95(64.6)$ & \\
\hline $\begin{array}{l}\text { Lymphatic } \\
\text { invasion }\end{array}$ & & & $<0.001$ \\
\hline Negative & 59 & $6(10.2)$ & \\
\hline Positive & 181 & 112 (61.9) & \\
\hline Venous invasion & & & $<0.001$ \\
\hline Negative & 81 & $13(16.0)$ & \\
\hline Positive & 159 & $105(66.0)$ & \\
\hline Liver metastasis & & & 0.178 \\
\hline Negative & 232 & $112(48.2)$ & \\
\hline Positive & 10 & $7 \quad(7.0)$ & \\
\hline $\begin{array}{l}\text { Peritoneal } \\
\text { dissemination }\end{array}$ & & & $<0.001$ \\
\hline Negative & 229 & $107(46.7)$ & \\
\hline Positive & 13 & $12(92.3)$ & \\
\hline Stage & & & $<0.001$ \\
\hline I & 87 & $17(19.5)$ & \\
\hline II & 41 & $22(53.7)$ & \\
\hline III & 59 & $33(55.9)$ & \\
\hline IV & 55 & $47(85.5)$ & \\
\hline
\end{tabular}

tive case. RPN2 expression was observed in the cytoplasm. RPN2 expression was observed in 119 (49.2\%) of 242 gastric adenocarcinoma patients.
RPN2 expression and clinicopathological factors in human gastric adenocarcinoma tissue. No relationships could be detected between RPN2 expression and the clinicopathological factors gender, histological type, and liver metastasis. However, expression was significantly higher in the cases with depth of wall invasion, lymph node metastasis, lymphatic invasion, venous invasion, peritoneal dissemination, and pathological stage (Table I).

Relationship between RPN2 expression and the histological stage of gastric adenocarcinoma. RPN2 expression was found in $17(19.5 \%)$ of 87 stage IA and IB gastric adenocarcinoma patients, $22(53.7 \%)$ of 41 stage IIA and IIB patients, $33(55.9 \%)$ of 59 stage IIIA, IIIB, and IIIC patients, and 47 (85.5\%) of 55 stage IV patients, indicating that the expression rate goes up with the advancement of stage (Table I).

Relationship between RPN2 expression and survival rate in all gastric adenocarcinoma patients. The 5-year survival rate was $89.9 \%$ in the gastric adenocarcinoma with no RPN2 expression in the primary lesion; whereas the 5-year survival rate of patients with RPN2 expression was significantly lower (29.8\%) (Fig. 2).

Relationship between RPN2 expression and recurrence rate by stage of gastric adenocarcinoma. The tumor recurrence rate among patients with stage II and III gastric adenocarcinoma was significantly higher for patients with RPN2 expression in the primary lesion compared to patients who did not express RPN2 (Fig. 3A and C). Also, the 5-year survival rate for stage II and III gastric adenocarcinoma patients with RPN2 expression-negative primary tumor was 100 and $80.4 \%$, whereas it was 38.7 and $46.9 \%$ for patients with RPN2 expression-positive tumors (Fig. 3B and D). Among patients with stage I gastric adenocarcinoma, no significant difference was observed in the recurrence rate between patients who expressed RPN2 in the primary lesion and those who did not (data not shown).

Clinicopathologic prognostic factors based on multivariate analysis. The factors found to differ significantly between RPN2-expressing and non-expressing patients by univariate analysis were examined by multivariate analysis. Histological type, peritoneal dissemination and RPN2 expression were determined to be clinicopathologic prognostic factors. The risk rate for RPN2 expression is shown in Table II.

Generation of RPN2 knockout cell lines by CRISPR/ Cas-mediated genome editing. In human gastric cancer cell lines, MKN45, MKN 74, KATO III and NUGC3 cells RPN2 expression was detected by RT-PCR methods (data not shown). For human gastric adenocarcinoma cell lines, MKN74 and KATO III, deficiency for RPN2 gene developed. We used the CRISPR/Cas9 technology to knockout RPN2 gene. Transformed and control cells for RPN2-KO-MKN74 (i.e., MKN74 transfected with a sgRPN2-expressing vector) RPN2-KO-KATO III (i.e., KATO III transfected with a sgRPN2-expressing vector) and control MKN74 and KATO III (empty vector transfected cells) were sorted based on the positive expression of the GFP reporter gene (Fig. 4B). RPN2 
A

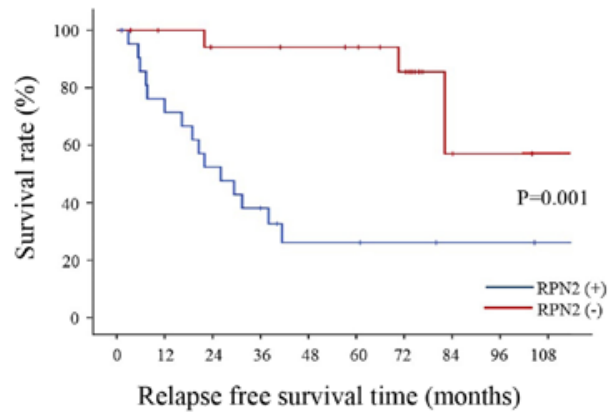

C

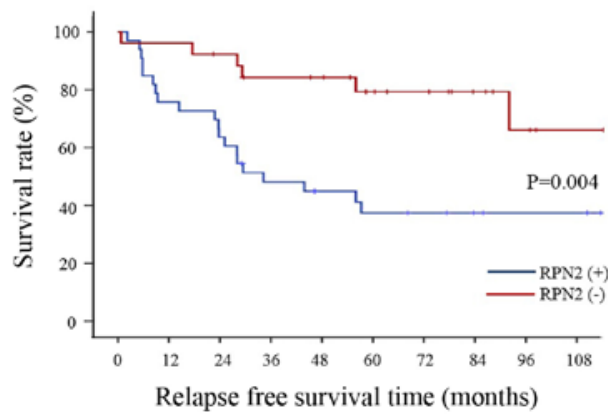

B

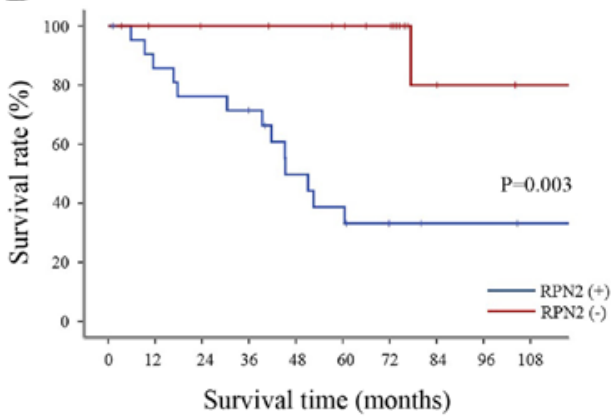

D

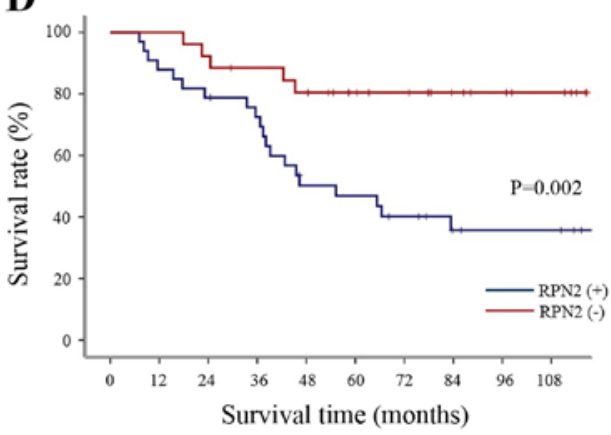

Figure 3. Relationship between RPN2 expression rates and survival rates and disease-free survival rates by stage of gastric adenocarcinoma patients. (A) Observed disease-free survival by stage II gastric cancer group. (B) Observed overall survival by stage II gastric cancer group. (C) Observed disease-free survival by stage III gastric cancer group. (D) Observed overall survival by stage III gastric cancer group. Stage II and III patients with RPN2-positive tumors had significantly poorer survival than those with RPN2-negative tumors.

$\mathbf{A}$

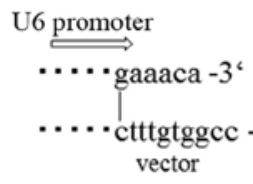

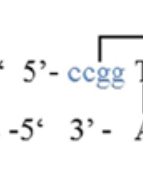
' TCTTCTCAGGCTCGCGGA
AGAAGAGACCGAGCGCC Insert (target sequence)

B
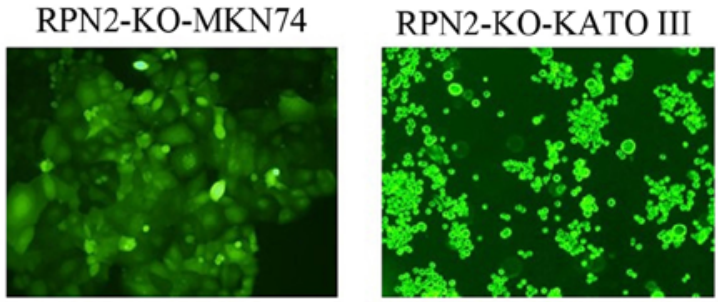

\section{$\mathbf{E}$}

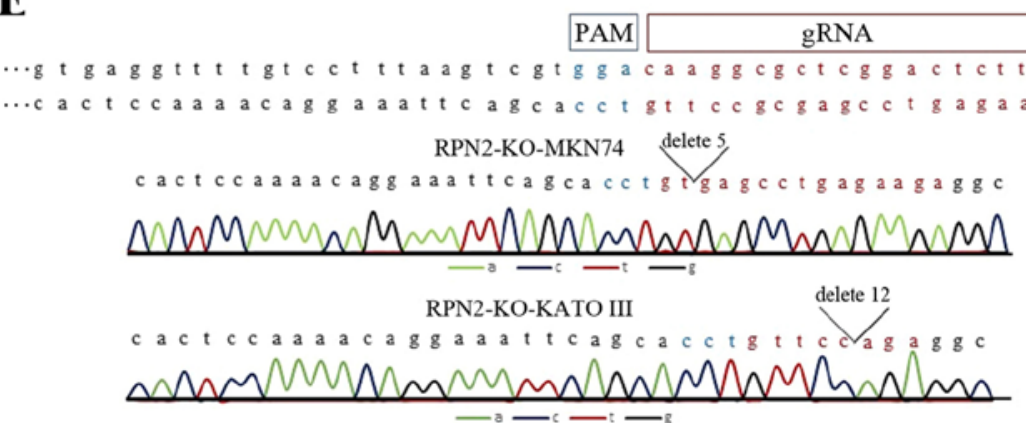

sgRNA

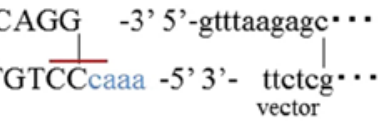
vector

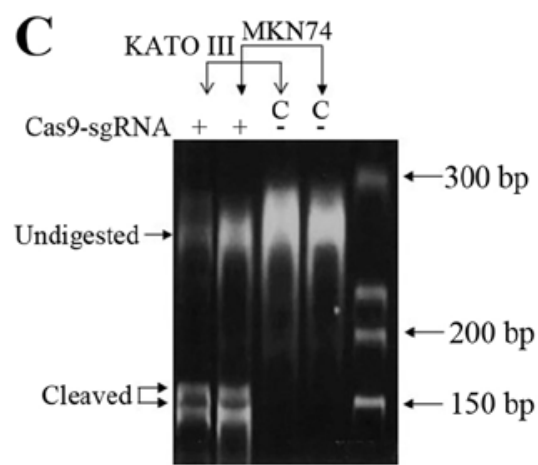

Indels (\%) 2135

D

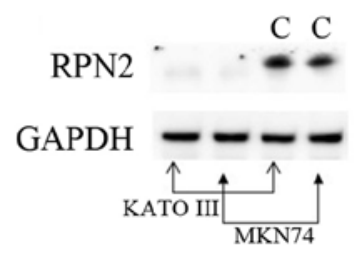

Figure 4. Detection of genome editing at RPN2 gene. (A) Cloning target RPN2 sequence using the Guide-it CRISPR/Cas9 system. A red line under AGG indicates protospacer-adjacent motif. (B) Fluorescence analysis shows that transfection with RPN2-Cas9-GFP plasmid increased GFP-expressing cells in MKN74 and KATO III cell lines. Fluorescence cell imaging were visualized $96 \mathrm{~h}$ following transfection. (C) Indel frequency was measured using the T7E1 assay and the percentages are shown at the bottom of each lane. (D) Protein extracted from fluorescence positive cells was analyzed for RPN2 and GAPDH by western blot assay. (E) Sanger sequencing of PCR products around gRNA binding site (red letters) and protospacer adjacent motif (PAM) site (blue letters). Wild-type reference sequences are given on the top. 
Table II. Pathological findings and RPN2 as prognostic factor for gastric cancer patients.

\begin{tabular}{lccccc}
\hline & \multicolumn{2}{c}{ Univariate analysis } & & Multivariate analysis \\
\cline { 2 - 3 } & Hazard ratio (95\% CI) & P-value & & Hazard ratio (95\% CI) & P-value \\
\hline Gender & $1.155(0.746-1.788)$ & 0.519 & & & \\
RPN2 & $10.540(5.849-18.991)$ & $<0.001$ & & $5.509(2.959-10.258)$ & $<0.001$ \\
Histological type & $1.862(1.236-2.804)$ & 0.03 & & $1.935(1.236-3.030)$ & 0.004 \\
Serosal invasion & $3.442(2.237-5.295)$ & $<0.001$ & & $1.234(0.759-2.007)$ & 0.397 \\
Lymphatic invasion & $20.478(5.040-83.200)$ & $<0.001$ & & $2.118(0.369-12.173)$ & 0.400 \\
Venous invasion & $10.590(4.623-24.258)$ & $<0.001$ & & $2.328(0.826-6.547)$ & 0.109 \\
Lymph node invasion & $5.332(2.963-9.594)$ & $<0.001$ & & $1.908(1.021-3.567)$ & 0.043 \\
Peritoneal dissemination & $5.309(2.645-10.656)$ & $<0.001$ & & $4.957(2.366-10.382)$ & $<0.001$ \\
Liver metastasis & $5.316(2.876-9.825)$ & $<0.001$ & & $1.520(0.772-2.993)$ & $<0.225$ \\
\hline
\end{tabular}

Table III. Correlation between RPN2 and p53 expression in gastric adenocarcinoma.

\begin{tabular}{lccr}
\hline & \multicolumn{2}{c}{ RPN2 expression } & \\
\cline { 2 - 3 } p53 expression & Negative & Positive & P-value \\
\hline Negative & 92 & 39 & \\
Positive & 31 & 80 & $<0.001$ \\
\hline
\end{tabular}

knockout was validated using the T7E1 assay. One guide used was efficient in knocking-out RPN2 gene in gastric adenocarcinoma cell lines. The Indel percentage from T7E1 assay was estimated at 21 and 35\%, for RPN2-KO KATO III and RPN2-KO MKN74 cells (Fig. 4C). Moreover, protein extracts were analyzed for RPN2 expression. The significant expression of RPN2 was undetected both in RPN2-KO MKN74 and KATO III cells, compared to respective intense bands in empty vector-transfected cells (Fig. 4D). We showed the Sanger-sequence data of RPN2-KO-MKN74 and RPN2-KOKATO III cells to prove CRISPR/Cas9 worked correctly (Fig. 4E). Chromatogram traces of RPN2-KO-MKN74 cells and RPN2-KO-KATO III cells showed the sequence of deletion mutations.

Knockout of RPN2 expression reduced the invasion ability of gastric adenocarcinoma cells. Fig. 5 demonstrates the cell invasion. While the number of invasion of MKN74 cells was 97 on average, the number of invasive RPN2-KO MKN74 cells was 13 on average. Similarly, while the mean number of invasive KATO III cells was 87 , the mean number of invasive RPN2-KO KATO III cells was 18.

Relationship between RPN2 and mutant p53. The expression of p53 was analyzed in the same samples of gastric adenocarcinoma. Eighty of 242 primary tumors showed positive immunoreactivity for $\mathrm{p} 53$; immunoreactivity was detected in the nuclei of carcinoma cells (Fig. 6A). Significant association was found between RPN2 immunostaining and p53 immunostaining $(\mathrm{p}<0.001)($ Table III). The patients with tumors expressing both RPN2 and p53 concomitantly was associated with higher depth of wall invasion, lymph node metastasis, lymphatic invasion, venous invasion, and pathological stage (Table IV). We show the number of invasion of MKN45 cells and MKN45 cells which were suppressed by RPN2 siRNA (Fig. 6B). There was no significant difference in invasive ability between MKN45 cells and MKN45 cells, which were suppressed by siRNA (Fig. 6C).

\section{Discussion}

In East Asia, gastric adenocarcinoma is one of the most common malignancies. In spite of the improvement in surgical treatment and chemotherapy, gastric adenocarcinoma of an advanced stage is still subject to a poor prognosis, although cases of early stage are successfully controlled. The risk factors for mortality among tumors are metastasis and drug resistance, and several reports describe the factors related to the invasiveness of malignant tumors and patient prognosis $(14,15)$. The human RPN2 gene examined in this study is positioned on chromosome 20q12-13.1, a region that is frequently deleted in patients with myeloid malignancies $(16,17)$. The RPN2 protein is a component of an N-oligosaccharyl transferase complex that conjugates high mannose oligosaccharides to asparagine residues in the $\mathrm{N}-\mathrm{X}-\mathrm{S} / \mathrm{T}$ consensus motif of nascent polypeptide chains $(18,19)$. RPN2 was reported as a key player of drug resistance by modulating glycosylation of multidrug resistance protein and, in vivo delivery of siRNA specific for RPN2 markedly reduced tumor growth in breast cancer (20). Also, downregulation of RPN2 efficiently induced apoptosis in the presence of docetaxel, and RPN2 promoted EMT through stabilization of mutant p53 in breast cancer $(12,20)$. Recent studies suggested that the acquisition of drug resistance by cancer cells might be modulated via the changes in miRNA levels and miRNAs play critical role in EMT of cancer cells (21-23).

In this study, RPN2 expression was found in the primary lesion of $49.2 \%$ of human gastric adenocarcinoma resection cases. In the RPN2 expression cases, the prevalence of clinicopathologic events was high, such as depth of wall invasion, lymph node metastasis and peritoneal dissemination, and asso- 


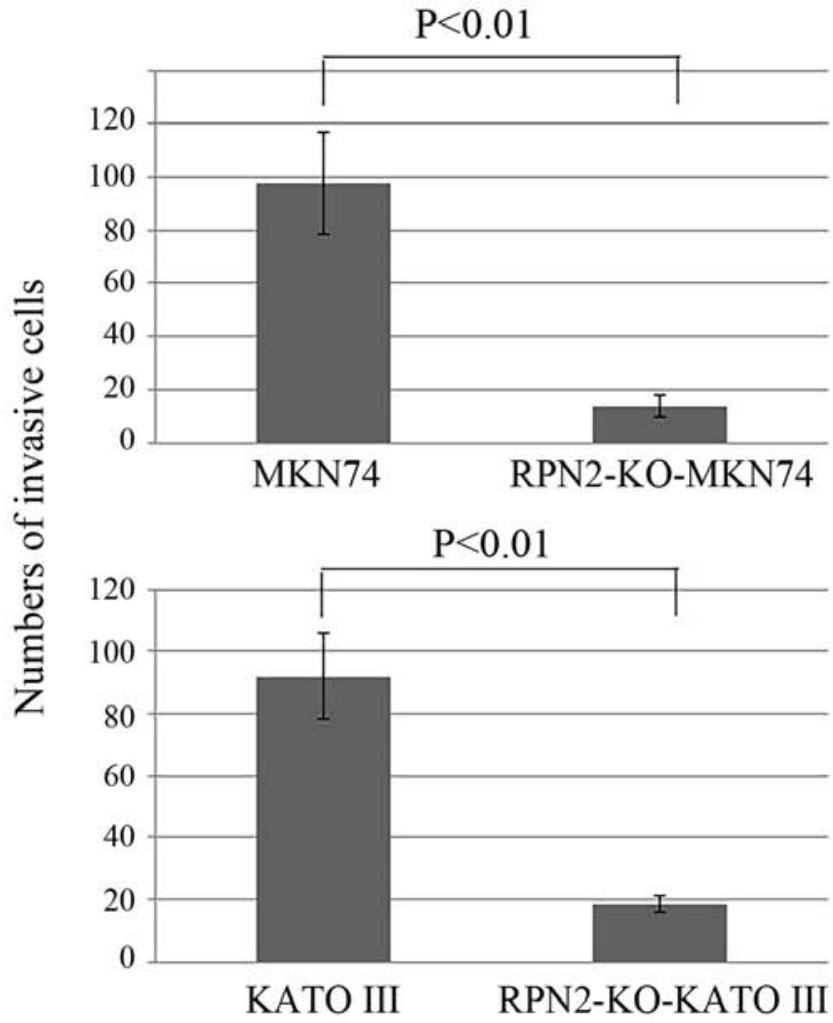

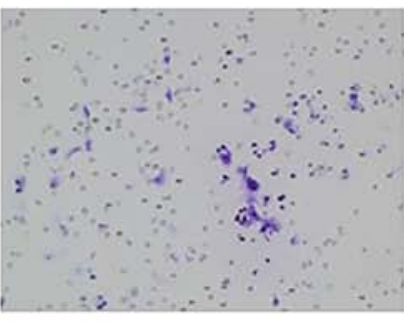

MKN74

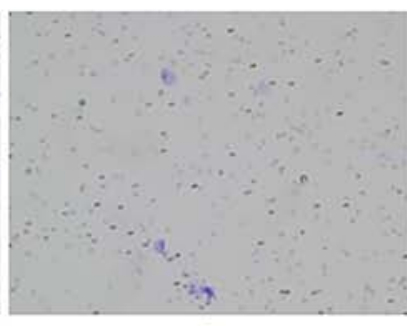

RPN2-KO-MKN74

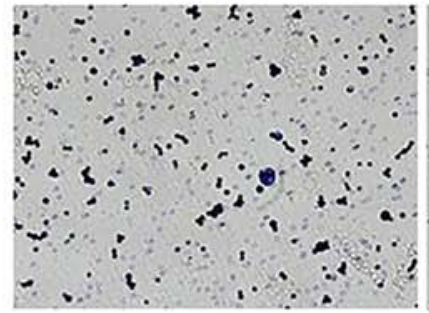

KATO III

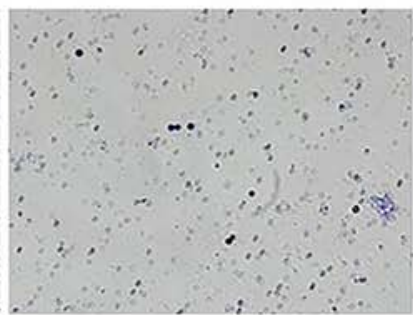

RPN2-KO-KATO III

Figure 5. Invasion assay in RPN2-KO-MKN74 and RPN2-KO-KATOIII cells. The knockout of RPN2 expression resulted in marked decline of invasion potential in MKN74 and KATO III human gastric cancer cell lines $(\mathrm{P}<0.01)$. Data were expressed as mean values \pm SD from triplicate experiments.

A

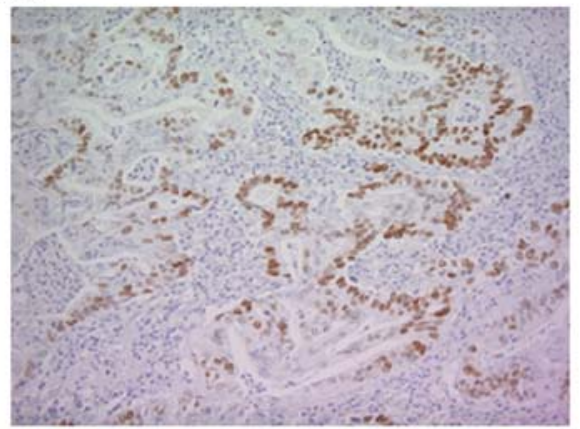

B

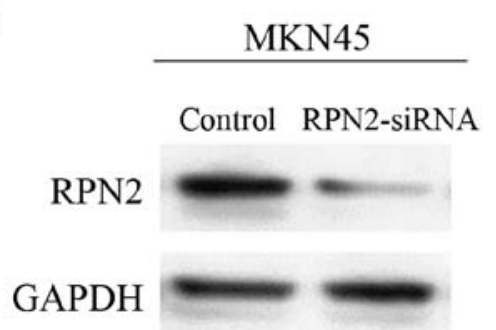

C
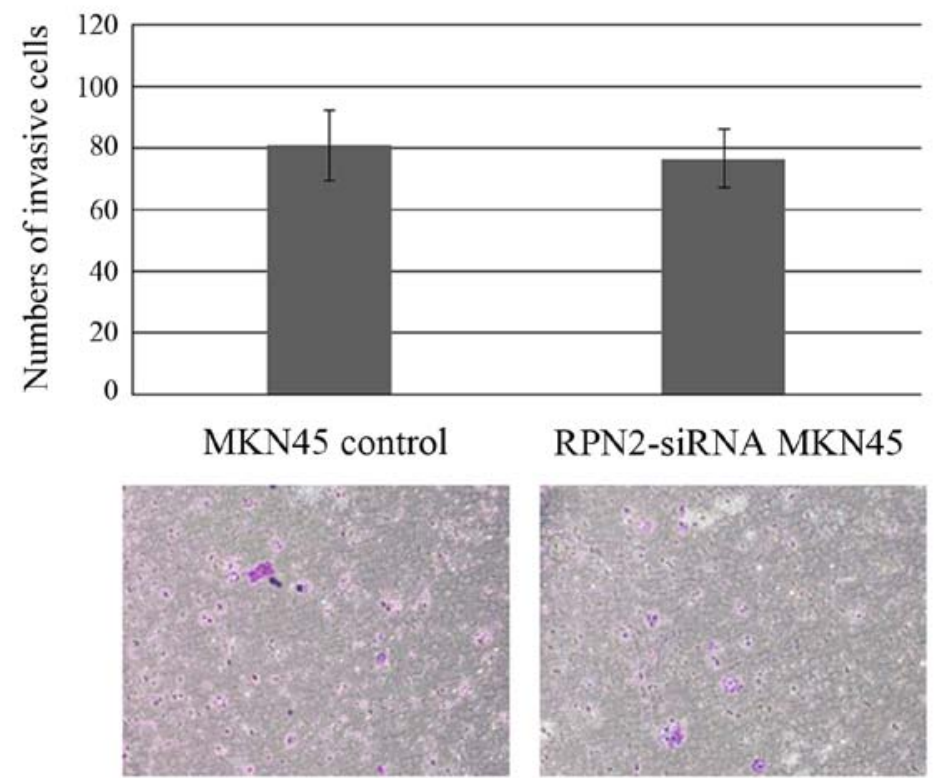

Figure 6. Relationship between RPN2 and p53 in gastric adenocarcinoma. (A) p53 protein stained in brown was detected in the nucleus of primary gastric adenocarcinoma lesions. (B) Evidence for knockdown of RPN2 caused by transfection with siRNA. MKN45 cells were transfected with scrambled siRNA (control); GAPDH was used as loading control. RPN2 siRNA followed by western blot assays using anti-RPN2 antibodies. (C) The knockout of RPN2 expression in MKN45 cell line did not reduce the ability of invasion. Data are expressed as mean values \pm SD from triplicate experiments.

ciated with poor patient prognosis. RPN2 expression was also identified as an independent prognosis factor. Furthermore, we showed correlation between RPN2 expression and mutant- type p53 expression in the primary lesion, and patients with tumors expressing both RPN2 and p53 concomitantly were associated with depth of wall invasion, lymph node metas- 
Table IV. Correlation of the expression of RPN2 and p53 concomitantly and clinicopathological findings.

\begin{tabular}{|c|c|c|c|}
\hline & \multirow[b]{2}{*}{ No. of cases } & \multirow{2}{*}{$\frac{\text { RPN2-positive }}{\text { No. of cases (\%) }}$} & \multirow[b]{2}{*}{ P-value } \\
\hline & & & \\
\hline All cases & 242 & $80(33.1)$ & \\
\hline Gender & & & 0.384 \\
\hline Male & 162 & $57(35.2)$ & \\
\hline Female & 80 & $23(28.8)$ & \\
\hline Histological & & & \\
\hline type & & & 0.785 \\
\hline Pap, tub & 128 & $41(32.0)$ & \\
\hline Por, sig & 111 & $37(33.3)$ & \\
\hline Muc & 3 & $2(66.7)$ & \\
\hline $\begin{array}{l}\text { Depth of wall } \\
\text { invasion }\end{array}$ & & & 0.001 \\
\hline $\mathrm{T} 1$ & 79 & $7 \quad(8.9)$ & \\
\hline $\mathrm{T} 2$ & 30 & $14(46.7)$ & \\
\hline $\mathrm{T} 3$ & 26 & $14(53.8)$ & \\
\hline $\mathrm{T} 4$ & 107 & $45(42.1)$ & \\
\hline $\begin{array}{l}\text { Lymph node } \\
\text { metastasis }\end{array}$ & & & 0.001 \\
\hline No & 95 & $15(15.8)$ & \\
\hline N1-3 & 147 & $65(44.2)$ & \\
\hline $\begin{array}{l}\text { Lymphatic } \\
\text { invasion }\end{array}$ & & & 0.001 \\
\hline Negative & 59 & $4 \quad(6.8)$ & \\
\hline Positive & 181 & $76(42.0)$ & \\
\hline Venous invasion & & & 0.001 \\
\hline Negative & 81 & $10(12.3)$ & \\
\hline Positive & 159 & $70(44.0)$ & \\
\hline Liver metastasis & & & 0.734 \\
\hline Negative & 232 & $76(32.8)$ & \\
\hline Positive & 10 & $4 \quad(4.0)$ & \\
\hline $\begin{array}{l}\text { Peritoneal } \\
\text { dissemination }\end{array}$ & & & 0.365 \\
\hline Negative & 229 & $74(32.3)$ & \\
\hline Positive & 13 & $6(46.2)$ & \\
\hline Stage & & & 0.001 \\
\hline I & 87 & $11(12.6)$ & \\
\hline II & 41 & $17(41.5)$ & \\
\hline III & 59 & $25(42.4)$ & \\
\hline IV & 55 & $27(49.1)$ & \\
\hline
\end{tabular}

tasis, lymphatic invasion, venous invasion, and pathological stage. The knockout of RPN2 expression reduced the ability of invasion in gastric adenocarcinoma cell lines MKN74 and KATO III. However, the knockout of RPN2 expression in MKN45 cell line did not reduce the ability of invasion. MKN74 and KATO III cell lines have mutant-type p53, and MKN45 cell line has wild-type p53 (24,25). RPN2 stabilized mutant-type $\mathrm{p} 53$ by suppressing glycogen synthase kinase $3 \beta$ in breast cancer cells (12). Additionally, RPN2 also regulated Bax and Bcl-2 in intrinsic apoptosis control (26). We believe that the invasive potential of gastric adenocarcinoma cells greatly affected the stabilization of mutant-type p53 by RPN2. Furthermore, the fact that the prognosis of RPN2-expressing gastric adenocarcinoma patients was poor was considered to involve not only the stabilized mutant-type p53 by RPN2 but also the control of p53-independent apoptosis pathway by RPN2.

This study has some limitations that require consideration. We showed that the expression of RPN2 may be involved in gastric adenocarcinoma cell invasion. However, we did not yet demonstrate the relationship between the RPN2 expression and EMT, which allows to increase motility and invasiveness of cancer cells (27). We intend to intensively explore the relationship of the RPN2 expression and oncogenic signal pathways including p53, which induce EMT. In addition, we think also there is a possibility that miRNAs are involved in the relation between EMT and RPN2 expression, because the expression of miRNAs including miR-200 family which repressed ZEB1/2 was controlled by p53 (28). In future, we will examine more deeply the intrinsic roles of RPN2 on the metastasis of gastric adenocarcinoma cells, and we want to make clear that RPN2 has the potential to be a new therapeutic target factor.

\section{Acknowledgements}

The technical assistance of Ms.M. Saito with this research is appreciated.

\section{References}

1. Dicken BJ, Bigam DL, Cass C, Mackey JR, Joy AA and Hamilton SM: Gastric adenocarcinoma: Review and considerations for future directions. Ann Surg 241: 27-39, 2005.

2. Nissan A, Garofalo A and Esquivel J: Cytoreductive surgery and hyperthermic intra-peritoneal chemotherapy (HIPEC) for gastric adenocarcinoma: Why haven't we reached the promised land? J Surg Oncol 102: 359-360, 2010.

3. Glehen O, Mohamed F and Gilly FN: Peritoneal carcinomatosis from digestive tract cancer: New management by cytoreductive surgery and intraperitoneal chemohyperthermia. Lancet Oncol 5: 219-228, 2004.

4. Calin GA and Croce CM: MicroRNA signatures in human cancers. Nat Rev Cancer 6: 857-866, 2006.

5. Bartel DP: MicroRNAs: Genomics, biogenesis, mechanism, and function. Cell 116: 281-297, 2004.

6. Ambros V: The functions of animal microRNAs. Nature 431: 350-355, 2004.

7. Takei Y, Takigahira M, Mihara K, Tarumi Y and Yanagihara K: The metastasis-associated microRNA miR-516a-3p is a novel therapeutic target for inhibiting peritoneal dissemination of human scirrhous gastric cancer. Cancer Res 71: 1442-1453, 2011.

8. Motoyama K, Inoue H, Mimori K, Tanaka F, Kojima K, Uetake H, Sugihara K and Mori M: Clinicopathological and prognostic significance of PDCD4 and microRNA-21 in human gastric cancer. Int J Oncol 36: 1089-1095, 2010.

9. Ueda T, Volinia S, Okumura H, Shimizu M, Taccioli C, Rossi S, Alder H, Liu CG, Oue N, Yasui W, et al: Relation between microRNA expression and progression and prognosis of gastric cancer: A microRNA expression analysis. Lancet Oncol 11: 136-146, 2010. 
10. Zhang Z, Liu S, Shi R and Zhao G: miR-27 promotes human gastric cancer cell metastasis by inducing epithelial-to-mesenchymal transition. Cancer Genet 204: 486-491, 2011.

11. Okugawa Y, Inoue Y, Tanaka K, Kawamura M, Saigusa S, Toiyama Y, Ohi M, Uchida K, Mohri Y and Kusunoki M: Smad interacting protein 1 (SIP1) is associated with peritoneal carcinomatosis in intestinal type gastric cancer. Clin Exp Metastasis 30: 417-429, 2013.

12. Takahashi RU, Takeshita F, Honma K, Ono M, Kato K and Ochiya T: Ribophorin II regulates breast tumor initiation and metastasis through the functional suppression of GSK3 $\beta$. Sci Rep 3: $2474,2013$.

13. Japanese Gastric Cancer Association: Japanese gastric cancer treatment guidelines 2010 (ver. 3). Gastric Cancer 14: 113-123, 2011.

14. Siegel R, Naishadham D and Jemal A: Cancer statistics, 2012. CA Cancer J Clin 62: 10-29, 2012.

15. Nguyen DX, Bos PD and Massagué J: Metastasis: From dissemination to organ-specific colonization. Nat Rev Cancer 9: 274-284, 2009.

16. Löffler C, Rao VV and Hansmann I: Mapping of the ribophorin II (RPN II) gene to human chromosome 20q12-q13.1 by in-situ hybridization. Hum Genet 87: 221-222, 1991.

17. Testa JR, Kinnealey A, Rowley JD, Golde DW and Potter D Deletion of the long arm of chromosome 20 [del(20)(q11)] in myeloid disorders. Blood 52: 868-877, 1978.

18. KelleherDJ,Kreibich GandGilmore R: Oligosaccharyltransferase activity is associated with a protein complex composed of ribophorins I and II and a 48 kd protein. Cell 69: 55-65, 1992.

19. Kelleher DJ and Gilmore R: An evolving view of the eukaryotic oligosaccharyltransferase. Glycobiology 16: 47R-62R, 2006.

20. Honma K, Iwao-Koizumi K, Takeshita F, Yamamoto $Y$, Yoshida T, Nishio K, Nagahara S, Kato K and Ochiya T: RPN2 gene confers docetaxel resistance in breast cancer. Nat Med 14: 939-948, 2008
21. Chen QY, Jiao DM, Wang J, Hu H, Tang X, Chen J, Mou H and Lu W: miR-206 regulates cisplatin resistance and EMT in human lung adenocarcinoma cells partly by targeting MET. Oncotarget 7: 24510-24526, 2016

22. Zhao JJ, Chu ZB, Hu Y, Lin J, Wang Z, Jiang M, Chen M, Wang X, Kang Y, Zhou Y, et al: Targeting the miR-221-222/PUMA/BAK/ BAX pathway abrogates dexamethasone resistance in multiple myeloma. Cancer Res 75: 4384-4397, 2015.

23. Ma J, Fang B, Zeng F, Ma C, Pang H, Cheng L, Shi Y, Wang H, Yin B, Xia J, et al: Down-regulation of miR-223 reverses epithelial-mesenchymal transition in gemcitabine-resistant pancreatic cancer cells. Oncotarget 6: 1740-1749, 2015

24. Yukimoto K, Nakata B, Muguruma K, Yashiro M, Ohira M, Ishikawa T, Hino M and Hirakawa K: Apoptosis and thymidylate synthase inductions by 5 -fluorouracil in gastric cancer cells with or without p53 mutation. Int J Oncol 19: 373-378, 2001.

25. Endo F, Nishizuka SS, Kume K, Ishida K, Katagiri H, Ishida K, Sato K, Iwaya T, Koeda K and Wakabayashi G: A compensatory role of NF- $\mathrm{KB}$ to $\mathrm{p} 53$ in response to 5-FU-based chemotherapy for gastric cancer cell lines. PLoS One 9: e90155, 2014.

26. Fujita Y, Yagishita S, Takeshita F, Yamamoto Y, Kuwano K and Ochiya T: Prognostic and therapeutic impact of RPN2-mediated tumor malignancy in non-small-cell lung cancer. Oncotarget 6: 3335-3345, 2015

27. Boyer B, Vallés AM and Edme N: Induction and regulation of epithelial-mesenchymal transitions. Biochem Pharmacol 60: 1091-1099, 2000

28. Kim T, Veronese A, Pichiorri F, Lee TJ, Jeon YJ, Volinia S, Pineau P, Marchio A, Palatini J, Suh SS, et al: p53 regulates epithelial-mesenchymal transition through microRNAs targeting ZEB1 and ZEB2. J Exp Med 208: 875-883, 2011. 